Mitteilungen der Österreichischen Geographischen Gesellschaft, 159. Jg. (Jahresband), Wien 2017, S. 413-415

\title{
Christine Embleton-Hamann zum 65. Geburtstag
}

\author{
Margreth KEILER, Bern, und Sven FuCHS, Wien* \\ mit 1 Abb. im Text
}

Im Februar 2018 feiert Christine Embleton-Hamann ihren 65. Geburtstag und wir nehmen dies zum Anlass, ihre wissenschaftlichen Arbeiten und ihr Engagement für die Geomorphologie in Österreich und auf internationaler Ebene zu würdigen. Christine EMBLETON-HAMANN ist bereits im März 2015 in ihren - wie sie es bezeichnet - „neuen Zeitabschnitt der selbstbestimmten Forschung“ (Pensionierung) übergetreten.

Christine Embleton-Hamann ist in Linz aufgewachsen, hat an der Universität Salzburg die Fächer Deutsch und Geographie studiert und im Jahr 1982 mit ihrer Dissertation zu „Buckelwiesen und Konvergenzformen am Südrand des Tennengebirges und in anderen Arealen der Nördlichen Kalkalpen“ promoviert. Ebenfalls an der Universität Salzburg wurde ihr die venia für Geographie verliehen. Bereits 1979 wechselte sie beruflich an das Institut für Geographie und Regionalforschung der Universität Wien, wurde 1994 zur Assistenzprofessorin und 1997 zur außerordentlichen Universitätsprofessorin ernannt.

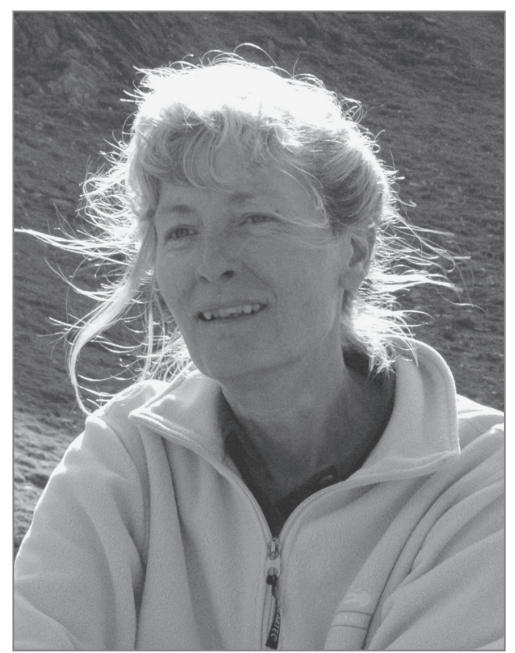

Christine Embleton-HamanN

\footnotetext{
* Prof. Dr. Margreth KeILER, Geographisches Institut der Universität Bern, Hallerstraße 12, CH-3014 Bern, Schweiz; E-Mail: margreth.keiler@giub.unibe.ch; PD Dr. Sven Fuchs, Institut für Alpine Naturgefahren, Universität für Bodenkultur, Peter-Jordan-Straße 82, A-1190 Wien, E-Mail: sven.fuchs@boku.ac.at
} 
In der Forschung befasst sich Christine EMBLETON-HAMAnN mit Themen der angewandten Physischen Geographie mit einem Schwerpunkt auf der Geomorphologie. Besonderes Interesse hat sie - neben ihren Arbeiten im europäischen Alpenraum - an Untersuchungen, die den Konnex zwischen Geomorphologie und globalen Umweltveränderungen herstellen. Von Beginn an spielten für sie Fragestellungen der (heute wieder aktuellen) Mensch-Umwelt-Beziehung eine bedeutende Rolle, vor allem in Hinblick auf die Entstehung spezifischer geomorphologischer Formen durch anthropogene Einwirkungen.

Wie wahrscheinlich vielen anderen auch ist uns der Name von Christine (Embleton-)HamanN zum ersten Mal in unserer eigenen Einführungsvorlesung zur Geomorphologie und auf den zugehörigen Exkursionen zu den Buckelwiesen bei Mittenwald begegnet. Während dieser Lehrveranstaltungen wurden die unterschiedlichen Ansätze zur Genese dieser besonderen Geländeform diskutiert. Die fundierten Grundlagen hierzu finden sich in der Dissertation der Jubilarin. Aufgrund der innovativen Erklärungsansätze zur Genese von Buckelwiesen wurden ihr 1982 der Johann-Hampel-Preis und ein Preis der Landesregierung Salzburg verliehen. In Folge hat Christine Embleton-Hamann seit den 1990er Jahren die Untersuchungen des Phänomens Buckelwiesen auf andere Gebiete ausgedehnt, mit einem Fokus auf Massenbilanzansätzen und der Frage nach einem anthropogenen Einfluss auf ihre Genese über die Verhinderung von Wiederbewaldung (EMBLETon-HAMANn 2004a; ECKER \& EMBLETON-HAMANN 2014).

Weitere Themen, die Christine Embleton-Hamann während ihrer beruflichen Laufbahn beschäftigten, sind Gefahrenprozesse und Risiken (z.B. EMBLETon-Hamann 1997) oder auch die Karmorphometrie und der glaziale Formenschatz (Embleton \& Hamann 1988; Embleton-Hamann 2004b). Hier arbeitet sie - aufgrund der neuartigen Forschungsergebnisse - in engem Kontakt mit Ian Evans von der Universität Durham. Die Anwendung geomorphologischer Methoden in der Landschaftsbewertung wurde während des Man-and-Biosphäre-Projekts in Obertauern vorangetrieben (EMBLETon-Hamann 1994).

Ihre Arbeiten zur Landschaftsbewertung und ihr umfassendes geomorphologisches Wissen führten auch zu ihrer langjährigen Tätigkeit als Gutachterin für das World Heritage Panel der International Union for Conservation of Nature (IUCN). Einen wesentlichen Beitrag leistet sie in Bezug auf Vergleiche von Konzepten in der deutschsprachigen und anglophonen Forschung. Ihre diesbezüglichen Publikationen (Embleton-Hamann \& Slaymaker 2006) gaben oft vergessenen Konzepten wieder die entsprechende Beachtung, auch für aktuelle Forschungsansätze in der Geomorphologie. Eine besondere Stellung nimmt hierbei das Thema der globalen Umweltveränderungen und deren Wirkung auf geomorphologische Prozesse ein. Das bemerkenswerte Buch „Geomorphology and Global Envrionmental Change“, welches im Rahmen der gleichnamigen Arbeitsgruppe der International Association of Geomorphology entstand und von Christine EMBLETON-HAMANN und Kollegen herausgegeben wurde (Slaymaker, Spencer \& Embleton-Hamann 2009), ist bereits ein Standardwerk in der Geomorphologie. Ihre diversen Forschungsvorhaben führten sie zu Auslandsaufenthalten am King's College London, sowie zur Waseda University Tokyo, zur National University of Taiwan, und zur University of British Columbia, Vancouver. Jene Kolleginnen und Kollegen, die mit der Jubilarin zusammenarbeiten dürfen, heben ihre gut durchdachten und neuartigen Ideen, die solide aufgearbeiteten Informationen und hochqualitativen Beiträge hervor.

Christine EmblEton-Hamann ist eine begnadete Hochschullehrerin, die es stets verstand, die Neugierde bei den Studentinnen und Studenten zu wecken und sie für die Geomorphologie und den Alpenraum zu begeistern. Im vertiefenden Rahmen des Hauptstudiums hat sie ihr detailreiches Wissen weitergegeben, aufgrund des fachlichen Niveaus galten ihre Lehrveranstaltungen bei Generationen von Studentinnen und Studenten als sehr anspruchsvoll.

Christine Embleton-Hamann engagiert sich nachhaltig in der Scientific Community. Sie fungiert als Assoziierte Editorin in der Zeitschrift für Geomorphologie, wirkte mehrere Perioden in der 
Kommission für Naturgefahren der Internationalen Geographischen Union (IGU), war von 1997 bis 2001 Publication Officer der International Association of Geomorphology und baute die heutigen Strukturen der Österreichischen Forschungsgruppe für Geomorphologie und Umweltwandel der Österreichischen Geographischen Gesellschaft auf. Ihrem Engagement und Einsatz ist es zu verdanken, dass heute die österreichische und internationale Geomorphologie in engem Kontakt und Austausch stehen. Mit Übertritt in den Ruhestand hat sie die Herausforderung angenommen, in der Serie „World Geomorphological Landscapes“ den Band „Landschaften und Landformen in Österreich“" zu koordinieren.

Wir möchten aus einer sehr persönlichen Sicht Christine für ihr Engagement für den wissenschaftlichen Nachwuchs danken. Sie hat uns - und auch viele Kolleginnen und Kollegen - immer gefördert, uns bereitwillig ihr akademisches Netzwerk zur Verfügung gestellt und uns auch in schwierigen Zeiten auf dem oftmals steinigen Weg der universitären Karriere begleitet und ermuntert, dabei zu bleiben. Wir wünschen ihr für ihren neuen Zeitabschnitt der selbstbestimmten Forschung alles Gute und viel Erfolg.

\section{Literaturhinweise}

Ecker M., Embleton-Hamann Ch. (2014), Das Phänomen der Buckelwiesen in den Dolomiten: Verbreitung und mögliche Entstehungstheorien. Hamburg, Diplomica Verlag.

Embleton-Hamann Ch. (1994), The Role of Geomorphological Mapping in Scenery Appraisal. In: Proceedings of the National Science Council - Part c: Humanities and Social Sciences, 4/2, S. 231-245. Taiwan.

Embleton-Hamann Ch. (1997), Naturgefahren in Österreich. Ursachen, Verbreitung, Schäden und Schutzmaßnahmen. In: Mitteilungen der Österreichischen Geographischen Gesellschaft, 139, S. 197-230.

Embleton-Hamann Ch. (2004a), Processes Responsible for the Development of a Pit and Mound Microrelief. In: Catena, 57, 2, S. 175-188.

Embleton-Hamann Ch. (2004b): 5 entries. In: Goudie A. (Hrsg.), Encyclopedia of Geomorphology. London, Routledge: "Erratic", Bd. 1, S. 337; "Haldenhang", Bd. 1, S. 512; "Hanging Valley", Bd. 1, S. 513; "Proglacial Landform", Bd. 2, S. 810-813; "Roche Moutonnée”, Bd. 2, S. 870.

Embleton C., Hamann Ch. (1988), A comparison of cirque forms between the Austrian Alps and the Highlands of Britain. In: Zeitschrift für Geomorphologie, N.F. Suppl.-Bd. 70, S. 75-93.

Embleton-Hamann Ch., Slaymaker O. (2006), Classics in physical geography: JäckLI, H., 1957: Gegenwartsgeologie des bündnerischen Rheingebietes. In: Progress in Physical Geography, 30, 6, S. 779-783

Slaymaker O., Spencer T., Embleton-Hamann Ch. (Hrsg.) (2009), Geomorphology and Global Environmental Change. Cambridge, Cambridge University Press. 